\title{
A study of orientational ordering in a fluid of dipolar Gay-Berne molecules using density-functional theory
}

\author{
Szabolcs Varga ${ }^{\text {a) }}$ \\ Department of Chemical Engineering and Chemical Technology, Imperial College of Science, Technology, \\ and Medicine, Prince Consort Road, London SW7 2BY, United Kingdom \\ István Szalai \\ Department of Physics, University of Veszprém, PO Box 158, Veszprém, H-8201 Hungary \\ János Liszi \\ Department of Physical Chemistry, University of Veszprém, PO Box 158, Veszprém, H-8201 Hungary
}

George Jackson

Department of Chemical Engineering and Chemical Technology, Imperial College of Science, Technology, and Medicine, Prince Consort Road, London SW7 2BY, United Kingdom

(Received 3 December 2001; accepted 21 February 2002)

\begin{abstract}
We present a density-functional approach to describe the orientational ordering of nonpolar and dipolar Gay-Berne fluids. The first-order perturbation theory developed by Velasco et al. [J. Chem. Phys. 102, 8107 (1995)] for a Gay-Berne fluid is simplified and tested for molecules with a length to breath ratio of $\kappa=3$ and energy anisotropies of $\kappa^{\prime}=1,1.25,2.5$, and 5 . The theory is found to be in fair agreement with existing simulation data for the location of the isotopic-nematic phase transition, but it overestimates the vapor-liquid critical point of the fluid due to a description of the free energy at the mean-field level. The effect on the phase behavior of including a central longitudinal point dipole within the Gay-Berne molecule is studied using a correct treatment of the long-range dipolar contribution at the level of a second-order virial theory [B. Groh and S. Dietrich, Phys. Rev. E 50, 3814 (1994)]. For a given energy anisotropy of $\kappa^{\prime}=5$ and reduced dipole moment $\mu^{*}=0.5$ we search for a stable ferroelectric nematic phase by changing the length to breath ratio $\kappa$. We do not find any evidence of ferroelectric nematic ordering for $\kappa>1.5$; the system only exhibits vapor-liquid and isotropic-nematic phase transitions for these values of the aspect ratios. For a slightly elongated and oblate shaped potential (e.g., $\kappa=0.5$ ), regions of stable isotropicferroelectric nematic and nematic-ferroelectric nematic phase coexistences are observed. The results of the theory indicate that a ferroelectic nematic fluid phase may be stabilized with respect to the positional ordering in the fluid of oblate dipolar particles. Comparison are made, where appropriate, with the existing results of Monte Carlo simulations for dipolar Gay-Berne fluids (Rull and co-workers, Molec. Phys. 94, 439 (1998); J. Chem. Phys. 109, 9529 (1998)). (C) 2002 American Institute of Physics. [DOI: 10.1063/1.1469607]
\end{abstract}

\section{INTRODUCTION}

A fine balance between anisotropic repulsive, dispersive forces, and polar interactions is crucial in determining the phase behavior of liquid crystal materials. Although the seminal view of Onsager ${ }^{1}$ that the repulsive hard core of the molecule gives rise to orientationally ordered phases is now well established (e.g., see simulation studies for hard ellipsoids $^{2,3}$ and hard-spherocylinders ${ }^{4-7}$ ), the nature of the dispersive and polar interactions influence the precise structure that is observed. In addition, the presence of molecular polarity can also give rise to an orientationally ordered phase which is polar. The focus of this contribution is first to examine the effect of a central point dipole oriented along the axis of symmetry (longitudinal dipoles) on the liquid crystalline phase transitions of prolate and oblate molecules with

\footnotetext{
a) Author to whom correspondence should be addressed. Electronic mail: s.varga@ic.ac.uk
}

anisotropic attractive dispersive forces. A particular emphasis is placed on the possible stability of ferroelectric nematic phases in such systems, a subject which has given rise to a great deal of debate in recent years. It is hoped that an analysis of the behavior of our simple model system will provide an understanding of the behavior of real thermotropic liquid crystals.

The examination of the effect of polar interactions on the structure and thermodynamic properties of fluids has been the subject of numerous studies. In a recent review, Teixeira et al. ${ }^{8}$ present a detailed discussion of the work on classical fluids with dipolar interactions. The attention of the review is mainly on the apparent absence of a vapor-liquid transition and the possibility of polar phases in systems of spherical (or near spherical) particles with strong dipolar interactions. The precise position and orientation of the polar interactions for nonspherical molecules are also very important in determining the structure of liquid crystalline phases. The main conclusions of theoretical and simulation studies of dipolar me- 
sogens have also been summarized in a recent paper. ${ }^{9}$ The main effect of a central dipole is to stabilize the positionally ordered (layered) liquid crystalline phases such as the smectic A, while the effect on the isotropic-nematic transition appears to be small and in some cases unfavorable; for molecules with longitudinally oriented dipoles positioned near one end (terminal dipoles), the nematic phase is stabilized relative the smectic phase. ${ }^{9}$ The majority of simulation studies of prolate dipolar molecules lead to the conclusion that polar nematic (ferroelectric) phases are not found for such systems; in the case of spherical and, less controversially, of oblate molecules there is some evidence for stable ferroelectric phases. In view of the large number of studies in the area we will only make a specific mention of the contributions that are directly relevant to our current work on the GayBerne system; the reader is directed to the previous reviews for more details.

The simple model mesogen that we examine in this work is the Gay-Berne particle ${ }^{10}$ with a central longitudinal point dipole. The Gay-Berne model is the most widely studied molecule with uniaxial anisotropic shape and attractive interactions; it can be viewed as an anisotropic version of the Lennard-Jones interaction. The nonpolar Gay-Berne system has been showed to exhibit uniaxial nematic and positionally ordered liquid crystalline phases such as smectic A and B, depending on the precise values of the interaction parameters. The first simulation studies ${ }^{11,12}$ were for a specific form of the potential which was slightly different from the original Gay and Berne recipe chosen to represent a four-site Lennard-Jones molecule. This preliminary work indicated that stable nematic and smectic A phases can be found in systems interacting via the Gay-Berne potential. Shortly afterwards a more complete examination of the temperaturedensity boundaries for vapor-liquid equilibria and for nematic and smectic B liquid crystalline phase behavior was reported for the original Gay-Berne parameterization using Gibbs ensemble Monte Carlo $^{13}$ and thermodynamic integration. ${ }^{14} \mathrm{~A}$ large number of groups have now simulated the liquid crystal phases of uniaxial Gay-Berne systems. Most of the studies are for prolate molecules, ${ }^{11-31}$ where as well as describing the structure and bulk phase behavior for differing values of the energy and shape anisotropy, the dynamic, ${ }^{18,29}$ nonequilibrium, ${ }^{21,22}$ and elastic properties ${ }^{26,27}$ have also been simulated. As expected the main conclusion of this body of work is that the smectic phase is stabilized for systems with large side-by-side attractive interactions and also for molecules with large aspect ratios. The studies for Gay-Berne molecules with an oblate shape anisotropy are less numerous, ${ }^{32-37}$ in addition to the isotropic and discotic nematic phases, columnar positional ordering is also seen for systems with sufficiently large energy and shape anisotropies. The vapor-isotropic, vapor-nematic, and isotropicnematic interface has also be the focus of some attention in simulations of liquid crystal films of prolate Gay-Berne particles. ${ }^{38-43}$ Further work in the area of a generalized Gay-Berne potential for biaxial and chiral molecules, confined systems, and mixtures has also appeared, but we do not discuss this in our short survey as the conclusions do not relate to the uniaxial pure component Gay-Berne systems examined in our work. As far as a theoretical description of the liquid crystalline phase behavior of Gay-Berne molecules is concerned, comparatively fewer studies have been reported probably as a result of the complicated anisotropic form of the potential. ${ }^{44-50}$ In one of the first theoretical studies which dates back to the time of the first simulations of Gay-Berne mesogens, Tjipto-Margo and Sullivan ${ }^{44}$ used a mean-field perturbation theory with the attractive term treated as a truncated spherical-harmonic expansion to examine the vapor-liquid phase behavior and orientational ordering of the system; however, due to the crude nature of the approximations employed the theory failed to give an adequate description of the phase diagram. Density-functional theories (DFTs) such as the one proposed by Velasco et al. ${ }^{46}$ (which we employ in our paper) are more suited to a quantitative description of anisotropic liquid crystalline fluids. Velasco et al. ${ }^{46}$ used a Weeks, Chandler, and Andersen (WCA) perturbation scheme together with a scaled Onsager density-functional theory to provide a good description of the simulated vapor-liquid and fluid-nematic phase behavior, ${ }^{13,14,17}$ they made no treatment of the smectic phases in their original paper. In extensions of the approach to deal with positional ordering ${ }^{48,49}$ a nonlocal version of the DFT with a Tarazona weighted density approximation (WDA) was employed, and good agreement with the simulated smectic phase boundaries was found. To conclude we emphasize that a clear understanding of the phase behavior of nonpolar prolate and oblate Gay-Berne molecules is of prime importance in understanding the effect of dipolar interactions on the liquid crystalline transitions.

We now turn to the central remit of this paper which is an understanding of the orientational ordering in the dipolar Gay-Berne system. The results of computer simulations of prolate and oblate Gay-Berne molecules with dipoles placed in a variety of positions and orientations interactions have now been reported. The first simulation studies of dipolar Gay-Berne mesogens were by Satoh et al. ${ }^{51}$; prolate molecules with central point dipoles oriented along the principal axis (longitudinal) were studied by Monte Carlo simulation using the parameters of Luckhurst and co-workers. ${ }^{11,12} \mathrm{Al}$ though the isotropic-nematic transition was not found to be significantly affected by the incorporation of a dipolar interaction, the smectic A phase was stablized by the stronger side-by-side interaction, as one would expect. A calculation of the ferroelectric order parameters indicated that the system did not exhibit a polar phase even for relatively large values of the dipole moment, in line with what was found for dipolar hard spherocylinders. ${ }^{9}$ A more detailed examination of the liquid crystal boundaries for Gay-Berne molecules with a central longitudinal dipole indicated that the nematic phase is suppressed for sufficiently strong dipolar interactions in favor of smectic A layering below an isotropic-nematicsmectic A triple point temperature. ${ }^{52-55}$ Gay-Berne molecules with an off-center (terminal) dipole oriented along the principal axis (longitudinal) have been studied; ${ }^{54-58}$ as for the hard spherocylinders with terminal longitudinal dipoles, ${ }^{59}$ the nematic phase is now stabilized with respect to smectic layering with a corresponding shift of the nematicsmectic transition to higher temperatures and densities. The 
structure of the smectic phase for the Gay-Berne systems with terminal dipoles is particularly interesting: whereas a simple smectic A (monolayer) phase is found for the molecules with a central dipole, in the case of terminal dipoles a striped anti-ferrolectric bilayer structure of layers with local ferroelectric order was seen. ${ }^{57}$ Simulations have also been performed for prolate Gay-Berne systems with dipoles oriented perpendicular to the principal axis (transverse) placed at the center ${ }^{60-62}$ and the end $\mathrm{d}^{63-65}$ of the molecules. The main observations are similar to those of the previous dipolar Gay-Berne systems: the dipolar interactions have little effect on the isotropic-nematic phase transition although a marked stabilization of the smectic phase is seen. Although some tilted smectic phase were reported in the earlier work, ${ }^{60,61}$ these now appear to be the results of periodic effects of the box shape. ${ }^{64}$ The vortex and ring dipole ordering found for the smectic phases of hard spherocylinders with central transverse dipoles ${ }^{66}$ was not found for the Gay-Berne systems. Of more direct relevance to our work are the studies of dipolar Gay-Berne molecules with an oblate shape anisotropy, where, as for the nonpolar Gay-Berne systems, much fewer studies have been made. Zannoni and co-workers have presented simulation results for oblate Gay-Berne molecules with central longitudinal ${ }^{67}$ and central transverse ${ }^{68}$ dipoles. In the case of the oblate molecules with a central longitudinal dipole four different temperatures were examined at a fixed density corresponding to isotropic, nematic discotic, and columnar phases. ${ }^{67}$ The nematic phase was found to be nonpolar, but only a single density and temperature was examined; we shall return to this point when we discuss our results. A small degree of ferroelectric order was found for the columnar state; small ferroelectric domains of five or six molecules with the dipoles in a head-to-tail geometry were present in the columns, but an anti-ferroelectric arrangement of adjacent columns was seen. This is line with the observations for hard cut spheres and hard ellipsoids with central longitudinal dipoles. ${ }^{69-72}$ Interestingly, Patey and co-workers ${ }^{71,72}$ found that a ferroelectric nematic phase can be formed in such systems of dipolar oblate particles, as was found for dipolar hard-sphere fluids. ${ }^{69,73-75}$ One should note that theses systems have a large susceptibility and a small residual field (due to a different treatment of the simulated boundaries, for example) which can give rise to polar samples. The simulations of oblate Gay-Berne molecules with transverse central dipoles illustrate this point: ${ }^{68}$ in the case of a weak transverse external field, polar columnar phases can be stabilized imparting a biaxiality on the system.

It is now clear from the preceding dicussion that a large number of computer simulation studies have been made to examine the effect of dipolar interactions on the ordered phases of Gay-Berne molecules. To our knowledge no theoretical investigation of the liquid crystalline phase diagram of dipolar Gay-Berne systems has been made; some limited work has appeared on the structure and elastic properties of these systems. ${ }^{76,77}$ We examine the effect of the dipolar interactions on the isotropic-nematic transition of Gay-Berne mesogens with central longitudinal dipoles making comparisons with the simulation data where possible. We do not treat states with positional order at this stage, and examine only orientational ordering. The second and perhaps more interesting aim of the work is to examine the possible stability of ferroelectric nematic phases especially in the case of oblate shaped molecules.

Before we discuss the details of the theory and the particular features of the phase behavior of the dipolar GayBerne system, it is important to mention the conflicting views on the subject of polar fluid phases. A stable ferroelectric nematic phase for thermotropic mesogens still eludes experimentalists, although polar ordering has been found for specifically designed polymeric materials such as aromatic polyesters and lyotropic polypeptides $;{ }^{78}$ spontaneous polarization is of course common in tilted smectic phases of chiral molecules due to a breaking of symmetry in such structures. A good summary of the state of play in the area of polarity of achiral mesogens can be found in the review of Blinov ${ }^{79}$ (also see Ref. 9). The most widely studied model of ferroelectrics is the classical Heisenberg exchange potential (continuous Ising model) ${ }^{80}$ the problem with these approaches is that since they often involve lattice models the system is forced to have a frozen positional order. In one of the first theoretical examinations of polar nematic phases, PalffyMuhoray et al. ${ }^{81}$ used a general Hamiltonian treated with a mean-field (Maier-Saupe) theory to show that ferroelectricity may be possible in systems of dipolar oblate shaped particles; this is certainly consistent with the findings of computer simulations of dipolar hard oblate particles mentioned earlier. ${ }^{71,72}$ Mean-field studies are being used to understand the general features of ferrolectric phase behavior (e.g., see Ref. 82). In the case of dipolar hard molecules of prolate shape, density-functional theories using the hypernetted chain (HNC) and mean-spherical approximation (MSA) closures indicate that ferroelectric phases may be stable in such systems; ${ }^{83-85}$ note that in their first paper, Perera and Patey ${ }^{84}$ did not locate a stable polar nematic phase due to an improper treatment of the long-range contribution to the dipolar interactions which was then corrected. ${ }^{85}$ Studies for dipolar hard spherocylinders with an Onsager free energy functional also indicate the possibility of regions of ferroelectric order in the phase diagram. ${ }^{86}$ One should note that the stability of ferrolectric phases of dipolar prolate molecules has not been confirmed by the simulation studies (see Ref. 9 and references therein). The main difficulty in dealing with systems with point dipoles is the long-range nature of the interaction and the lack of screening (in contrast with what is found for charged systems). From a careful and rigorous treatment of the electromagnetic properties of systems with dipolar interactions, Groh and Dietrich ${ }^{87-92}$ have used a densityfunctional treatment with the correct second virial coefficient of the long-range dipolar interaction to show that the stability of the polar phases is governed both by the shape of the sample and the nature of the dielectric medium surrounding it. Their calculations for the spherical Stockmayer system $^{87-90,92}$ indicated that the system exhibits a ferroelectric fluid phase, although for small values of the dipole moment the polar fluid phase was shown to be preempted by a freezing transition. ${ }^{90}$ The numerous other theoretical studies of phase transitions in dipolar spherical particles are reviewed in Ref. 8. In the case of hard ellipsoids and sphero- 
cylinders with central longitudinal dipoles, the theory of Groh and Dietrich ${ }^{91}$ predicts that the nematic phase is stabilized by the polar interactions and that ferroelectric order is possible for systems of near-spherical or oblate shape. The latter feature is consistent with the findings of the simulations, ${ }^{69-75}$ although the freezing transitions have not been examined in the theory. Not everyone is convinced that polar order is possible in fluid phases. Indeed, Lagerwall ${ }^{93}$ has used symmetry arguments developed for solids to argue that polar ordering in fluids is unlikely; however, no treatment of the boundary conditions was incorporated into the analysis. This having been said we employ the approach of Groh and Dietrich to develop a density-functional theory and examine the ordering transitions (nematic and ferroelectric nematic) in systems of dipolar Gay-Berne molecules.

\section{DIPOLAR GAY-BERNE MODEL}

The dipolar Gay-Berne intermolecular pair potential model examined in this contribution consists of a short-range anisotropic term incorporating repulsive and dispersive attractive contributions [the usual Gay-Berne (GB) model], and a long-range dipolar term

$$
u=u^{\mathrm{GB}}+u^{\mathrm{Dip}} .
$$

The Gay-Berne interaction energy $\left(u^{\mathrm{GB}}\right)$ is the anisotropic version of the well-known Lennard-Jones potential and is given by

$$
\begin{aligned}
u^{\mathrm{GB}}\left(\underline{r}_{12}, \underline{\omega}_{1}, \underline{\omega}_{2}\right)= & 4 \varepsilon\left(\underline{\omega}_{12}, \underline{\omega}_{1}, \underline{\omega}_{2}\right) \\
& \times\left[\left(\frac{\sigma_{s s}}{r_{12}-\sigma\left(\underline{\omega}_{12}, \underline{\omega}_{1}, \underline{\omega}_{2}\right)+\sigma_{s s}}\right)^{12}\right. \\
& \left.-\left(\frac{\sigma_{s s}}{r_{12}-\sigma\left(\underline{\omega}_{12}, \underline{\omega}_{1}, \underline{\omega}_{2}\right)+\sigma_{s s}}\right)^{6}\right],
\end{aligned}
$$

where the unit vector $\underline{\omega}_{i}$ defines the orientation of the main axis of particle $i$. The vector connecting the centers of particles 1 and 2 is given as the product of a unit vector and the intermolecular distance $\left(\underline{r}_{12}=r_{12} \underline{\omega}_{12}\right)$. The depth of the potential energy well $\varepsilon\left(\underline{\omega}_{12}, \underline{\omega}_{1}, \underline{\omega}_{2}\right)$ depends on the orientational unit vectors, the length to breath ratio $\left(\kappa=\sigma_{e e} / \sigma_{s s}\right)$ and the energy depth anisotropy $\left(\kappa^{\prime}=\varepsilon_{s s} / \varepsilon_{e e}\right)$, which are defined as the ratio of the size and energy interaction parameters in the end-to- end and side-by-side configurations

$$
\begin{aligned}
\varepsilon\left(\underline{\omega}_{12}, \underline{\omega}_{1}, \underline{\omega}_{2}\right)= & \varepsilon_{s s}\left\{1-\chi^{2}\left(\underline{\omega}_{1} \underline{\omega}_{2}\right)^{2}\right\}^{-1 / 2} \\
& \times\left\{1-\frac{1}{2} \chi^{\prime}\left[\frac{\left(\underline{\omega}_{12} \underline{\omega}_{1}+\underline{\omega}_{12} \underline{\omega}_{2}\right)^{2}}{1+\chi^{\prime} \underline{\omega}_{1} \underline{\omega}_{2}}\right.\right. \\
& \left.\left.+\frac{\left(\underline{\omega}_{12} \underline{\omega}_{1}-\underline{\omega}_{12} \underline{\omega}_{2}\right)^{2}}{1-\chi^{\prime} \underline{\omega}_{1} \underline{\omega}_{2}}\right]\right\}^{2},
\end{aligned}
$$

where

$$
\chi=\frac{\kappa^{2}-1}{\kappa^{2}+1}
$$

and

$$
\chi^{\prime}=\frac{\kappa^{\prime 1 / 2}-1}{\kappa^{\prime 1 / 2}+1} .
$$

The distance $\sigma\left(\underline{\omega}_{12}, \underline{\omega}_{1}, \underline{\omega}_{2}\right)$ at which the potential vanishes is the distance of closest approach between two "hard Gaussian overlap" (HGO) particles

$$
\begin{aligned}
\sigma\left(\underline{\omega}_{12}, \underline{\omega}_{1}, \underline{\omega}_{2}\right)= & \sigma_{s s}\left\{1-\frac{1}{2} \chi\left[\frac{\left(\underline{\omega}_{12} \underline{\omega}_{1}+\underline{\omega}_{12} \underline{\omega}_{2}\right)^{2}}{1+\chi \underline{\omega}_{1} \underline{\omega}_{2}}\right.\right. \\
& \left.\left.+\frac{\left(\underline{\omega}_{12} \underline{\omega}_{1}-\underline{\omega}_{12} \underline{\omega}_{2}\right)^{2}}{1-\chi \underline{\omega}_{1} \underline{\omega}_{2}}\right]\right\}^{-1 / 2} .
\end{aligned}
$$

The full description of the Gay-Berne model can be found in the original paper, ${ }^{10}$ where the parameters $\kappa$ and $\kappa^{\prime}$ were set to 3 and 5 in order to provide a close representation of the pair potential between two four-site Lennard-Jones molecules. In this paper both parameters will be varied to examine their separate effects on the phase behavior.

The form of dipolar pair potential due to the embedded point dipole $(\mu)$ into the center of the particles along the main axis can be written as follows:

$$
u^{\operatorname{Dip}}\left(\underline{r}_{12}, \underline{\omega}_{1}, \underline{\omega}_{2}\right)=\left\{\begin{array}{l}
0, \quad r_{12}<\sigma\left(\underline{\omega}_{12}, \underline{\omega}_{1}, \underline{\omega}_{2}\right) \\
\frac{\mu^{2}}{r_{12}^{3}} w\left(\underline{\omega}_{12}, \underline{\omega}_{1}, \underline{\omega}_{2}\right), \\
\quad \text { for } r_{12} \geqslant \sigma\left(\underline{\omega}_{12}, \underline{\omega}_{1}, \underline{\omega}_{2}\right),
\end{array}\right.
$$

where

$$
w=\underline{\omega}_{1} \underline{\omega}_{2}-3\left(\underline{\omega}_{1} \underline{\omega}_{12}\right)\left(\underline{\omega}_{2} \underline{\omega}_{12}\right) .
$$

\section{PERTURBATION THEORY OF ISOTROPIC, NEMATIC, AND FERROELECTRIC PHASES}

\section{A. Gay-Berne system}

Before details of the theoretical treatment of the dipolar molecules are described, we outline the perturbation theory for the fluid of nonpolar Gay-Berne molecules. In the usual perturbation treatment we separate the interaction potential into a short ranged repulsive reference term and an attractive term

$$
u^{\mathrm{GB}}=u^{\mathrm{rep}}+u^{\mathrm{att}} .
$$

According to the perturbation theory of Weeks, Chandler, and Andersen (WCA), ${ }^{94}$ the repulsive part of the potential is obtained by shifting the potential with respect to the well depth of the interaction energy:

$$
u^{\mathrm{rep}}\left(\underline{r}_{12}, \underline{\omega}_{1}, \underline{\omega}_{2}\right)=\left\{\begin{array}{c}
u^{\mathrm{GB}}\left(\underline{r}_{12}, \underline{\omega}_{1}, \underline{\omega}_{2}\right)+\varepsilon\left(\underline{\omega}_{12}, \underline{\omega}_{1}, \underline{\omega}_{2}\right), \\
\quad \text { for } r_{12}<r_{m}\left(\underline{\omega}_{12}, \underline{\omega}_{1}, \underline{\omega}_{2}\right) \\
0, \quad \text { otherwise. }
\end{array}\right.
$$

The repulsive potential clearly remains anisotropic. Correspondingly, the attractive term is given by

$$
\begin{aligned}
u^{\text {att }}\left(\underline{r}_{12}, \underline{\omega}_{1}, \underline{\omega}_{2}\right) & \\
\quad= & \left\{\begin{array}{ll}
-\varepsilon\left(\underline{\omega}_{12}, \underline{\omega}_{1}, \underline{\omega}_{2}\right) & r_{12}<r_{m}\left(\underline{\omega}_{12}, \underline{\omega}_{1}, \underline{\omega}_{2}\right) \\
u^{G B}\left[\underline{r}_{12}, \underline{\omega}_{1}, \underline{\omega}_{2}\right] & r_{12} \geqslant r_{m}\left(\underline{\omega}_{12}, \underline{\omega}_{1}, \underline{\omega}_{2}\right)
\end{array} .\right.
\end{aligned}
$$

In the WCA prescription $r_{m}$ is the distance at which the potential takes its minimum value. The free energy of a system of particles interacting through a soft anisotropic repulsive potential such as (5) can be determined by using a per- 
turbation theory with the standard Barker-Henderson (BH) temperature dependent contact distance; for the present model, however, the contact distance also depends on the orientations of the particles. Instead of following the $\mathrm{BH}$ route we make the following approximation:

$$
\begin{aligned}
u^{\mathrm{rep}}\left(\underline{r}_{12}, \underline{\omega}_{1}, \underline{\omega}_{2}\right) & \approx u^{\mathrm{HGO}}\left(\underline{r}_{12}, \underline{\omega}_{1}, \underline{\omega}_{2}\right) \\
& =\left\{\begin{array}{cc}
\infty & r_{12}<\sigma\left(\underline{\omega}_{12}, \underline{\omega}_{1}, \underline{\omega}_{2}\right) \\
0 & \text { otherwise }
\end{array} .\right.
\end{aligned}
$$

In this way, the free energy functional of the Gay-Berne fluid is given as the sum of two contributions

$$
F^{\mathrm{GB}}[f]=F_{\text {ref }}^{\mathrm{HGO}}[f]+F_{\text {pert }}^{\text {att }}[f] .
$$

Here, the singlet orientational distribution function $f(\omega)$ gives the functional dependence of the free energy on the molecular orientation, and will be made explicit later in this section. The first term corresponds to the free energy of the reference HGO model, which in the language of the theory of Onsager ${ }^{1,95}$ can be written as

$$
F_{\text {ref }}^{\mathrm{HGO}}[f]=F_{\text {id }}[f]+F_{\text {exc }}[f] .
$$

This contribution incorporates the ideal translational and orientational entropy terms, and is given by

$$
\frac{\beta F_{\mathrm{id}[}[f]}{N}=\ln \left(\Lambda^{3} \rho\right)-1+\int f(\underline{\omega}) \ln (4 \pi f(\underline{\omega})) d \underline{\omega},
$$

where $\beta=1 / k T$ ( $T$ is the temperature, and $k$ is the Boltzmann constant), $N$ is the number of particles, $\Lambda$ is the de Broglie wave length, and $\rho=N / V$ is the number density ( $V$ is the volume).

The simplest representation of the excess (residual) free energy contribution due to the intermolecular interactions is that of Onsager, which is exact at the level of the second virial coefficient (excluded volume) term, and as a consequence is only valid in the low-density limit (where the ordering transitions occur for systems with infinite aspects ratios). There are a number of ways of extending the adequacy of the Onsager theory to systems with realistic aspect ratios (see the review of Vroege and Lekkerkerker ${ }^{95}$ for details). Approaches range from the early work with scaled particle theory (SPT), ${ }^{96}$ through the prescription of scaled virial expansions (e.g., see Refs. 97-99), to the use of DFTs originally developed for inhomogeneous systems. ${ }^{100}$ The most successful description of the ordering transitions in hard convex-body models are based on the standard decoupling approximation of Parsons. ${ }^{101,102}$ This approximation allows us to separate the spatial dependence of the excluded volume from its orientational dependence by a suitable scaling to an equivalent hard-sphere (HS) model. In the simplest implementation of the decoupling approximation for our system, one only requires a knowledge of the second virial coefficients for the HGO and HS potentials with the same molecular volume, together with an expression for the free energy of the hard-sphere fluid

$$
\frac{\beta F_{\mathrm{exc}}[f]}{N}=\frac{\beta F_{\mathrm{exc}}^{\mathrm{HS}}}{N} \frac{B_{2}^{\mathrm{HGO}}[f]}{B_{2}^{\mathrm{HS}}} .
$$

Such an expression can be derived following a number of routes, and we refer the reader to the original papers for further details. In the particular case of interest there is no spatial dependence on the repulsive contribution to the free energy as we are only concerned with orientationally ordered and disordered fluid phases. By using the Carnahan and Starling ${ }^{103}$ expression for the residual free energy of the equivalent $\mathrm{HS}$ of the same volume together with the exact expression for the second virial coefficient of the HGO particle, ${ }^{104,105}$ the excluded volume contribution to the free energy of the HGO fluid can be written as

$$
\begin{aligned}
\frac{\beta F_{\text {exc }}[f]}{N}= & \frac{4 \eta-3 \eta^{2}}{(1-\eta)^{2}} \iint \sqrt{\frac{1-\chi^{2}\left(\underline{\omega}_{1} \underline{\omega}_{2}\right)^{2}}{1-\chi^{2}}} \\
& \times f\left(\underline{\omega}_{1}\right) f\left(\underline{\omega}_{2}\right) d \underline{\omega}_{1} d \underline{\omega}_{2},
\end{aligned}
$$

where the packing fraction is defined in terms of the volume of the HGO molecule by $\eta=\rho \nu^{\mathrm{HGO}}$. The packing fraction is related to the reduced density usually employed in simulation studies by $\eta=\kappa \pi \rho^{*} / 6$ where $\rho^{*}=\rho \sigma_{s s}^{3}$.

At the level of the first-order perturbation theory, the contribution of the attractive interactions to the free energy functional has the form

$$
\begin{aligned}
F_{\text {pert }}^{\text {att }}[f]= & \frac{1}{2} \rho^{2} \iiint \int u_{a t t}\left(\underline{r}_{12}, \underline{\omega}_{1}, \underline{\omega}_{2}\right) g^{\mathrm{HGO}}\left(\underline{r}_{12}, \underline{\omega}_{1}, \underline{\omega}_{2}\right) \\
& \times f\left(\underline{\omega}_{1}\right) f\left(\underline{\omega}_{2}\right) d \underline{r}_{1} d \underline{r}_{2} d \underline{\omega}_{1} \underline{\omega}_{2} .
\end{aligned}
$$

Here, the pair correlation function $g^{\mathrm{HGO}}\left(\underline{r}_{12}, \underline{\omega}_{1}, \underline{\omega}_{2}\right)$ of the reference HGO system has to be determined, which is a complicated function of the orientations of the two molecules and intermolecular distance. This is a formidable computational task not only for the ordered phases but even for the homogeneous isotropic phase. One way of overcoming this problem is to use of the previously mentioned decoupling approximation for the pair correlation function as described by Velasco et al. ${ }^{46}$ Due to the complex form of the Gay-Berne potential, however, this still involves a substantial computational burden. In order to keep the expression as manageable as possible, a simple ad hoc mean-field approximation is used, which incorporates the main properties of the firstorder perturbation theory for the Lennard-Jones fluid. ${ }^{106} \mathrm{In}$ essence the approximation involves setting the pair correlation function $g(r)$ to unity and at the same time truncating the radial integral at a cut-off distance of $r_{c}=2.5 \sigma$. This choice gives a vapor-liquid phase boundary that is in very good agreement with simulation results for the LennardJones system. Additionally, it provides a very good representation of density profile for an inhomogeneous LennardJones fluid near a single hard wall. ${ }^{107}$ In the current study we extend this treatment to a Gay-Berne fluid with an orientational dependent cutoff $r_{c}=2.5 \sigma\left(\underline{\omega}_{12}, \underline{\omega}_{1}, \underline{\omega}_{2}\right)$, and write the attractive perturbation to the free energy of the Gay-Berne system as

$$
\begin{aligned}
\beta F_{\text {pert }}^{\mathrm{att}}[f] / N= & \frac{1}{2} \rho^{*} \beta \iiint \hat{u}\left(\underline{\omega}_{12}, \underline{\omega}_{1}, \underline{\omega}_{2}\right) \\
& \times f\left(\underline{\omega}_{1}\right) f\left(\underline{\omega}_{2}\right) d \underline{\omega}_{12} d \underline{\omega}_{1} d \underline{\omega}_{2},
\end{aligned}
$$


where

$\hat{u}\left(\underline{\omega}_{12} \underline{\omega}_{1}, \underline{\omega}_{2}\right)=\int_{0}^{2.5 \sigma^{*}\left(\underline{\omega}_{12}, \underline{\omega}_{1}, \underline{\omega}_{2}\right)} u^{a t t}\left(x, \underline{\omega}_{12}, \underline{\omega}_{1}, \underline{\omega}_{2}\right) x^{2} d x$.

In this expression the quantities are scaled in terms of the cross-section diameter for the side-by-side configuration $\left(\sigma_{s s}\right): x=r_{12} / \sigma_{s s}$ and $\sigma^{*}\left(\underline{\omega}_{12}, \underline{\omega}_{1}, \underline{\omega}_{2}\right)=\sigma\left(\underline{\omega}_{12}, \underline{\omega}_{1}, \underline{\omega}_{2}\right) /$ $\sigma_{s s}$. Using the following notation for the integral of the attractive energy over the relative orientations:

$$
\zeta=\frac{1}{\varepsilon_{s s}} \int \hat{u}\left(\underline{\omega}_{12}, \underline{\omega}_{1}, \underline{\omega}_{2}\right) d \underline{\omega}_{12},
$$

we can write the attractive contribution to the free energy functional as

$$
\begin{aligned}
\beta F_{\text {pert }}^{\mathrm{att}}[f] / N= & \frac{1}{2} \frac{\rho^{*}}{T^{*}} \iiint \zeta\left(\underline{\omega}_{1} \underline{\omega}_{2}\right) \\
& \times f\left(\underline{\omega}_{1}\right) f\left(\underline{\omega}_{2}\right) d \underline{\omega}_{1} d \underline{\omega}_{2},
\end{aligned}
$$

where the reduced temperature is defined as $T^{*}=1 / \beta \varepsilon_{s s}$.

\section{B. Dipolar Gay-Berne system}

Now that we have outlined the theory of the orientationally ordered Gay-Berne fluid, we extend the treatment to the dipolar Gay-Berne system. The dipolar contribution is treated as an additional perturbation to the free energy functional

$$
F^{\mathrm{DGB}}[f]=F_{\text {ref }}^{\mathrm{HGO}}[f]+F_{\text {pert }}^{\text {att }}[f]+F_{\text {pert }}^{\mathrm{Dip}}[f] .
$$

At the level of second virial theory, the dipolar perturbation term can be written as

$$
\beta F_{\text {pert }}^{\text {Dip }}[f] / N=\rho B_{2}^{\text {Dip }}[f],
$$

where the second virial coefficient is given by

$$
\begin{aligned}
B_{2}^{\operatorname{Dip}}[f]= & -\frac{1}{2 V} \iiint \int f_{M}\left(\underline{r}_{12}, \underline{\omega}_{1}, \underline{\omega}_{2}\right) f\left(\underline{\omega}_{1}\right) \\
& \times f\left(\underline{\omega}_{2}\right) d \underline{r}_{1} d \underline{r}_{2} d \underline{\omega}_{1} d \underline{\omega}_{2},
\end{aligned}
$$

and the Mayer function of the dipole-dipole interaction is

$f_{M}\left(\underline{r}_{12}, \underline{\omega}_{1}, \underline{\omega}_{2}\right)$

$$
=\left\{\begin{array}{l}
0, \quad r_{12}<\sigma\left(\underline{\omega}_{12}, \underline{\omega}_{1}, \underline{\omega}_{2}\right) \\
\exp \left(-\beta u^{\operatorname{Dip}}\left(\underline{r}_{12}, \underline{\omega}_{1}, \underline{\omega}_{2}\right)\right)-1, \quad \text { otherwise }
\end{array} .\right.
$$

It has been shown ${ }^{108,109}$ that the use of Eq. (18), which is sometimes referred to as a modified mean-field approximation, results in a better description of the vapor-liquid coexistence curve for polar fluids than the usual mean-field theory. The Taylor series expansion of the Mayer function is given by

$$
f_{M}=\sum_{n=1}^{\infty} \frac{\left(-\beta u^{\text {Dip }}\right)^{n}}{n !} .
$$

The substitution of the first term of Eq. (20) into Eq. (19) results in an expression to first order in the dipolar potential

$$
\begin{aligned}
B_{2}^{\mathrm{Dip}, \mathrm{LR}}[f]= & \frac{1}{2 V} \iiint \int \beta u^{\operatorname{Dip}}\left(\underline{r}_{12}, \underline{\omega}_{1}, \underline{\omega}_{2}\right) \\
& \times f\left(\underline{\omega}_{1}\right) f\left(\underline{\omega}_{2}\right) d \underline{r}_{1} d \underline{r}_{2} d \underline{\omega}_{12} d \underline{\omega}_{1} d \underline{\omega}_{2} .
\end{aligned}
$$

This expression represents the long-range contribution to the second virial coefficient of the dipolar system, with the potential decaying as $1 / r_{12}^{3}$. Due to convergence considerations, the spatial integration has to be performed carefully for such an expression. In a series of very interesting and thought provoking papers, Groh and Dietrich ${ }^{87-92}$ have considered this problem in detail, and have concluded that the shape of the fluid sample has to be considered explicitly with this type of integral. By performing the spatial integration in an uniaxial ellipsoidal sample with axial ratio $(k)$, one obtains the following result:

$$
\begin{aligned}
B_{2}^{\mathrm{Dip}, \mathrm{LR}}[f]= & -\frac{\beta \mu^{2}}{2} \iint f\left(\cos \theta_{1}\right) f\left(\cos \theta_{1}\right)\left(\widetilde{w}\left(\underline{\omega}_{1}, \underline{\omega}_{2}\right)\right. \\
& \left.+4 \pi I(k) \cos \theta_{1} \cos \theta_{2}\right) d \underline{\omega}_{1} d \underline{\omega}_{2},
\end{aligned}
$$

where

$$
\widetilde{w}\left(\underline{\omega}_{1}, \underline{\omega}_{2}\right)=\int w\left(\underline{\omega}_{1}, \underline{\omega}_{2}, \underline{\omega}_{12}\right) \ln \sigma\left(\underline{\omega}_{12}, \underline{\omega}_{1}, \underline{\omega}_{2}\right) d \underline{\omega}_{12} .
$$

The function $I(k)$ provides the shape dependence and is related to the depolarization factor $D(k): I(k)=1 / 3-D(k)$. It can be shown that in the limit of a spherical sample, $k=1$ and $I(k)=0$, but the depolarization factor is nonzero; this gives rise to a depolarization field in the bulk, which should be taken into account in any theory of dipolar systems. This effect is neglected in most theoretical treatments of ordering transitions in dipolar systems (e.g., see Refs. 99 and 110), and can lead to a misleading conclusion about the possible existence of a ferroelectric nematic phase. The other limit of particular interest is the infinitely thin and long ellipsoidal sample for which there is no depolarization effect

$$
I(k \rightarrow \infty)=\frac{1}{3} .
$$

The principal reason for using this ellipsoidal symmetry in Eq. (22) is that one obtains the correct shape independent thermodynamic limit for a system with homogeneous magnetization, as has been pointed out by Groh and Dietrich. ${ }^{89}$

The higher order terms of the Mayer function Eq. (20) result in a short-range contribution to the second virial coefficient Eq. (19), which does not cause convergence problems and can be integrated in the standard way

$$
\begin{aligned}
B_{2}^{\mathrm{Dip}, \mathrm{SR}}[f]= & -\frac{1}{2} \iiint \int \sum_{n=2}^{\infty} \frac{\left(-\beta u^{\mathrm{Dip}}\left(\underline{r}_{12}, \underline{\omega}_{1}, \underline{\omega}_{2}\right)\right)^{n}}{n !} \\
& \times f\left(\underline{\omega}_{1}\right) f\left(\underline{\omega}_{2}\right) r_{12}^{2} d r_{12} d \underline{\omega}_{12} d \underline{\omega}_{1} d \underline{\omega}_{2} .
\end{aligned}
$$

The use of $n=30$ terms is found to be more than sufficient to ensure convergence of the series. The dipole moment of the system is characterized in terms of a reduced value which is defined in the usual way as $\mu^{*}=\sqrt{\mu^{2} /\left(\varepsilon_{s s} \sigma_{s s}^{3}\right)}$. 


\section{Minimization of the free energy functional}

In order to calculate the free energy of the system, the equilibrium orientational distribution function has to be determined. This can be done by a functional minimization of the corresponding free energy functionals with respect to the orientation distribution function $f(\underline{\omega})$, i.e., Eq. (8) for the Gay-Berne fluid and Eq. (17) for the dipolar Gay-Berne fluid, with the constraint that $f(\underline{\omega})$ remains normalized, $\int f(\underline{\omega}) d \underline{\omega}=1^{95}$

$$
\left.\frac{\partial\left(\beta F[f] / N+\lambda\left(1-\int f(\underline{\omega}) d \underline{\omega}\right)\right)}{\partial f(\underline{\omega})}\right|_{f_{\mathrm{eq}}(\underline{\omega})}=0,
$$

where $\lambda$ is a Lagrange undetermined multiplier. By specifying the explicit form of the free energy functional, this Euler-Lagrange integral equation can be written in an integrated form which does not require an explicit knowledge of $\lambda$. This equation is solved by iteration following the procedure of Herzfield et al. ${ }^{111}$ with a slight modification. The polar angle $\theta$ is discretised in the range 0 to $\pi$, and a Simpson's quadrature is used to evaluate the integrals. The integrals over the relative molecular orientations $\underline{\omega}_{12}$ for the dispersive attractive interactions of Eq. (15), for the short-range dipolar interaction of Eq. (23), and for the sum of the longrange dipolar interactions given by Eq. (25) are expanded in terms of Legendre polynomials, ${ }^{95}$ to reduce the computational burden. Only a fourth-order expansion is necessary to get a reliable representation of the free energy for moderate elongations of the particles $(\kappa<3)$. It is important to note at this stage that the form of the single orientational distribution function $f(\underline{\theta})$ is not prescribed a priori, e.g., as a series of Legendre polynomials (see Ref. 95), and is not treated as symmetrical about $\theta=\pi / 2$; this allows us to describe ferroelectric nematic phases as well as the usual nematic phase.

Once the equilibrium orientational distribution function and free energy are known, the pressure $P=-(\partial F / \partial V)_{N, T}$ and the chemical potential $\mu=-(\partial F / \partial N)_{V, T}$ can be determined from the standard thermodynamic relations. The phase diagrams of the system can then be obtained by ensuring the equality of the chemical potentials, pressures, and temperatures in the coexisting phases. These phase coexistence conditions are solved numerically using a simplex method. ${ }^{112}$

In the present study, only the isotropic (vapor and liquid) and orientationally ordered (nematic and ferroelectric nematic) fluid phases are considered. These phases are characterized by the following ordered parameters: the usual nematic order parameter $S$ is used to quantify the ordering with respect to the nematic director chosen to lie along the $z$ axis

$$
\begin{aligned}
S & =\int P_{2}(\cos (\theta)) f(\underline{\omega}) d \underline{\omega} \\
& =\frac{1}{3} \int\left(3 \cos ^{2}(\theta)-1\right) f(\underline{\omega}) d \underline{\omega},
\end{aligned}
$$

where $P_{2}$ is the second Legendre polynomial. A value of $S$ $=0$ represents an orientationally disordered isotropic fluid phase, while a value of $S=1$ would correspond to a perfectly oriented phase (nematic or ferroelectric nematic). The nematic order parameter cannot be used to distinguish the polar

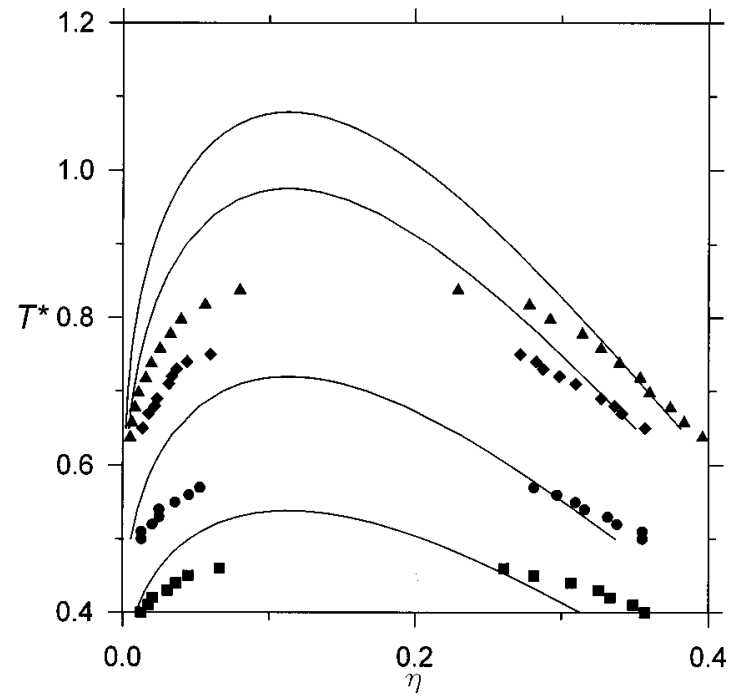

FIG. 1. The effect of the energy depth anisotropy $\left(\kappa^{\prime}\right)$ on the vapor-liquid coexistence of the Gay-Berne system with $\kappa=3$. The curves correspond to the results of the theory, and the points are the results of simulations (Ref. 27). From top to bottom $\kappa^{\prime}=1,1.25,2.5$, and 5 .

ferroelectic nematic order from the usual nematic order possessing up-down symmetry. A polarity parameter $M$, which is defined in terms of the first Legendre polynomial $P_{1}$, has the required property, since it is nonzero only for the ferroelectic phases

$$
M=\int P_{1}(\cos (\theta)) f(\underline{\omega}) d \underline{\omega}=\int \cos (\theta) f(\underline{\omega}) d \underline{\omega} .
$$

Although the values of the polar order parameter $M$ are not reported it allows us to distinguish between the nematic and ferroelectric nematic phases.

\section{RESULTS AND DISCUSSION}

\section{A. Gay-Berne system}

Our results for the fluid phase equilibria of the reference Gay-Berne fluid are presented first to demonstrate the reliability of approximations used in the theory. In Fig. 1 we show the effect of varying the energy anisotropy $\kappa^{\prime}$ on the vapor-liquid coexistence boundaries for systems with a fixed elongation $\kappa$. It can be seen that a decrease in the energy anisotropy promotes the vapor-liquid transition by shifting the phase envelope to higher temperatures. The decrease in the energy anisotropy leads to a larger contribution to the attractive interactions [see Eq. (2)], which are the driving force for the vapor-liquid transitions. The agreement between the theory and simulation results ${ }^{27}$ is satisfactory. As for all analytical descriptions of the free energy, the theory overestimates the vapor-liquid critical temperature; a renormalization group (RG) theory of the critical region is required for the correct treatment of the critical region, but this is beyond the scope of the current study. The added consequence is that the theory increasingly underestimates the coexisting vapor densities as the critical point is approached; the discrepancy for the saturated liquid densities is less marked. Despite this failure, the approximation used for the attractive contribution [Eq. (14)] provides a much better rep- 
resentation of the vapor-liquid equilibria than the usual mean-field approximation. ${ }^{44,46}$ We have also examined the effect of molecular elongation on the vapor-liquid coexistence curve. For a slightly larger elongation of $\kappa=3.2$, we find that the phase envelope is shifted to lower temperatures due to a decrease in the relative contribution to the attractive energy. This is consistent with the results of the simulations. ${ }^{28}$ The agreement between the theoretically predicted and simulated vapor-pressures is also satisfactory. We can conclude that approximation of Eq. (14) captures the main features of the attractive contribution of the potential and provides a good representation of the vapor-liquid equilibria of the Gay-Berne fluid.

We now turn to an examination of the orientationally ordered phases of the Gay-Berne system; as the molecules possess up-down symmetry, only the nematic phase is examined in this case. The equation of state and the nematic order parameter of the Gay-Berne model with $\kappa=3$ and $\kappa^{\prime}=5$ for a temperature $T^{*}=1.25$, which is above the vapor-liquid critical point are displayed in Fig. 2. The latest simulation study ${ }^{31}$ indicates that as the system is compressed there is a transition from an isotropic fluid phase (with a packing fraction of $\left.\eta_{I}=0.495\right)$ to an orientationally ordered nematic phase (with a packing fraction of $\eta_{N}=0.506$ and a nematic order parameter of $S=0.55$ ) at a pressure of about $P_{I-N}^{*}=\beta P v_{0}=8.17$. The theory underestimates the pressure close to the isotopic-nematic transition, but the location of the isotropic-nematic transition density is very close to the simulated value: the theoretical predictions for the densities of the coexisting isotropic and nematic phases are $\eta_{I}$ $=0.476$ and $\eta_{N}=0.487$ with a transition pressure of $P_{I-N}^{*}$ $=6.17$. The orientational ordering of the fluid is well represented by the theory, not only at the isotropic-nematic transition (with a predicted value of $S=0.53$ ) but also along the nematic branch of the isotherm as can be seen from the order parameter curve in Fig. 2. Since the repulsive anisotropic forces play the dominant role in the orientational ordering it will have become clear at this stage that the use of the decoupling approximation (11) together with the HGO reference system (7) provides the essential features of the repulsive contribution of the Gay-Berne potential.

\section{B. Dipolar Gay-Berne system}

In order to assess the adequacy of the theory in describing the dipolar interactions, we start by examining the vapor-liquid phase equilibria of the Stockmayer model (the dipolar Lennard-Jones system is equivalent to the GayBerne system with $\kappa=\kappa^{\prime}=1$ ). The theoretical predictions of the vapor-liquid coexistence envelope for the Stockmayer system are compared to the Gibbs ensemble simulation data of Smit et al. ${ }^{113}$ in Fig. 3 for various values of the reduced dipole moment $\mu^{*}=\sqrt{\mu^{2} / \varepsilon_{s s} \sigma_{s s}^{3}}=\sqrt{\mu^{2} / \varepsilon \sigma^{3}}$; the theory is seen to provide a good representation of the vapor-liquid equilibria for this system of dipolar spherical molecules, although it suffers from the same problems as the description of the nonpolar Gay-Berne systems (see Fig. 1).

We now come to the central goal of this contribution: An examination of the fluid phase behavior of Gay-Berne mol-
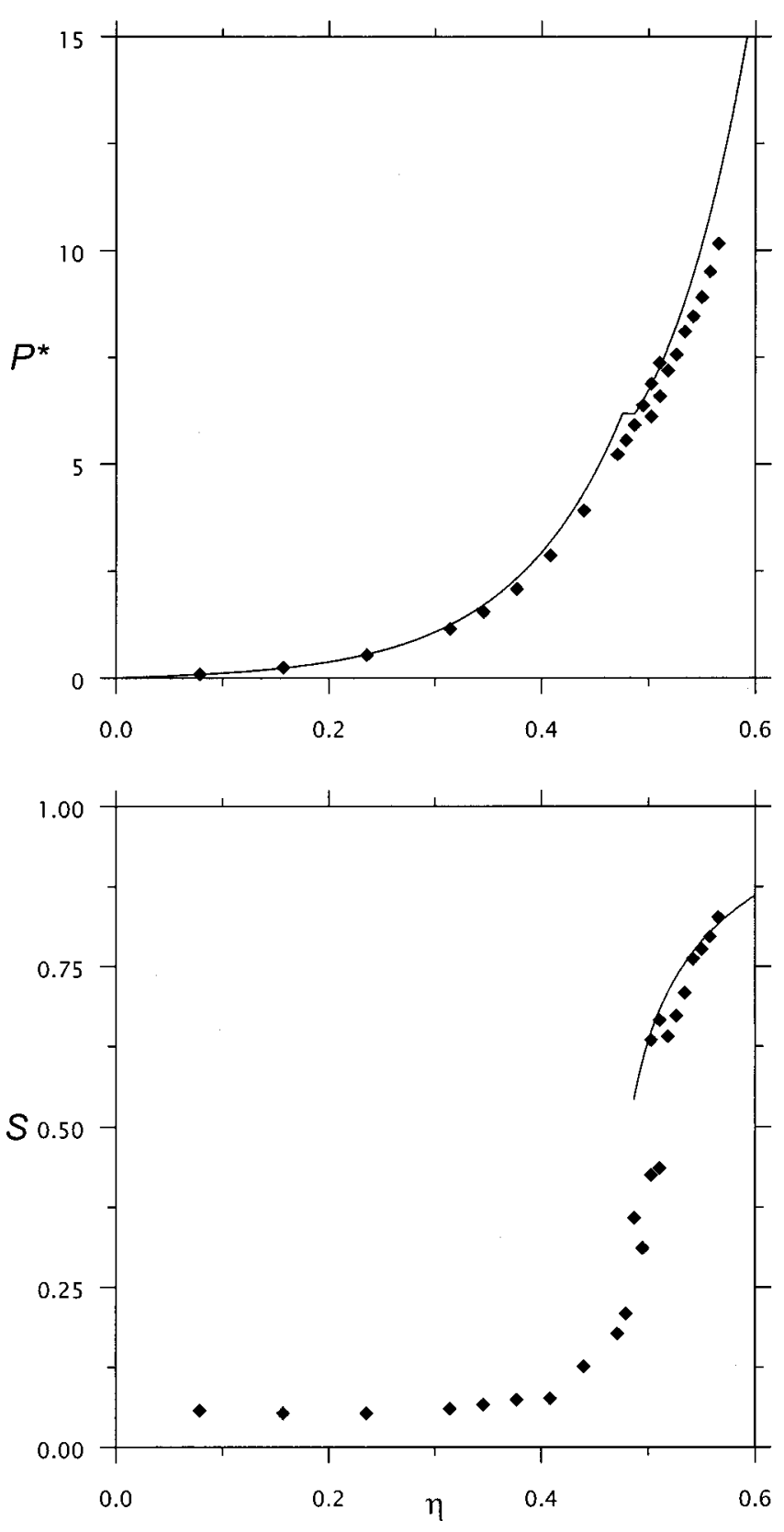

FIG. 2. Isotherm and nematic order parameter at $T^{*}=1.25$ for the GayBerne system with $\kappa=3$ and $\kappa^{\prime}=5\left(P^{*}=\beta P / \nu_{0}, \eta=\rho \nu_{0}\right)$. The curves correspond to the results of the theory, and the points are the results of simulations (Ref. 14).

ecules with central point dipoles oriented along the principal molecular axis. The orientational ordering of our dipolar Gay-Berne fluid is particularly intriguing, because the dipolar interaction breaks the up-down symmetry of the interaction energy. The dipolar interaction potential favors the parallel end-to-end and the antiparallel side-by-side configurations. The parallel end-to-end configuration is most dominant for the particles which are nearly spherical or possess oblate $(\kappa \gtrless 1)$ shapes, while the antiparallel configuration is more energetically favorable for the longer prolate particles $(\kappa>1)$. This results in a very subtle interplay between the shape of the particle and the dipolar interactions in the stabilization or destabilization of the orientationally ordered phases for different type of particle shapes. 


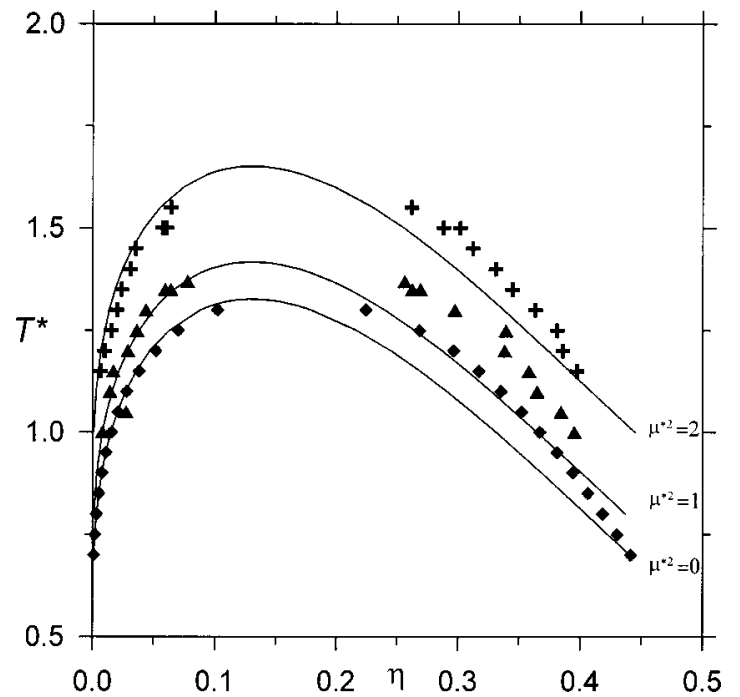

FIG. 3. Vapor-liquid equilibria for the Stockmayer system $\left(\kappa=1\right.$ and $\kappa^{\prime}$ $=1$ ) with dipole moments $\mu^{*}=\sqrt{\mu^{2} / \varepsilon \sigma^{3}}=0,1$, and 2 . The curves correspond to the results of the theory, and the points are the results of MC simulations (Ref. 113).

Before discussing the phase behavior of oblate shaped dipolar Gay-Berne molecules, we demonstrate the accuracy of the theory in describing the isotropic-nematic phase transitions for prolate Gay-Berne molecules with central longitudinal dipoles by comparing the theoretical predictions with the isothermal-isobaric MC simulation data of Rull and co-workers. ${ }^{52,53}$ In Figs. 4 and 5 we can see the effect of gradually increasing the dipole moment on the equation of state and on the nematic order parameter for dipolar GayBerne molecules of prolate shape $(\kappa=3)$; vapor-liquid equilibria are not seen as the system is in a state $\left(T^{*}\right.$
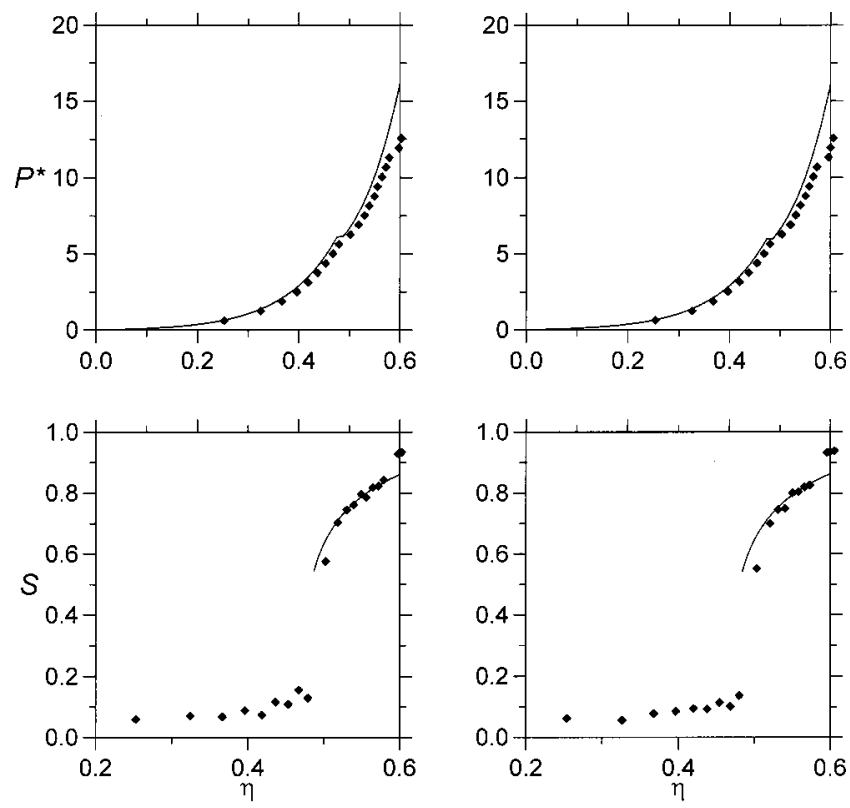

FIG. 4. Isotherm and nematic order parameter at $T^{*}=1.25$ for the dipolar Gay-Berne system with $\kappa=3$ and $\kappa^{\prime}=5$. The figures on the left are for a dipole moment of $\mu^{*}=0.5$, while the figures on the right are for $\mu^{*}=1$. The curves correspond to the results of the theory, and the points are the results of simulations (Refs. 52 and 53).
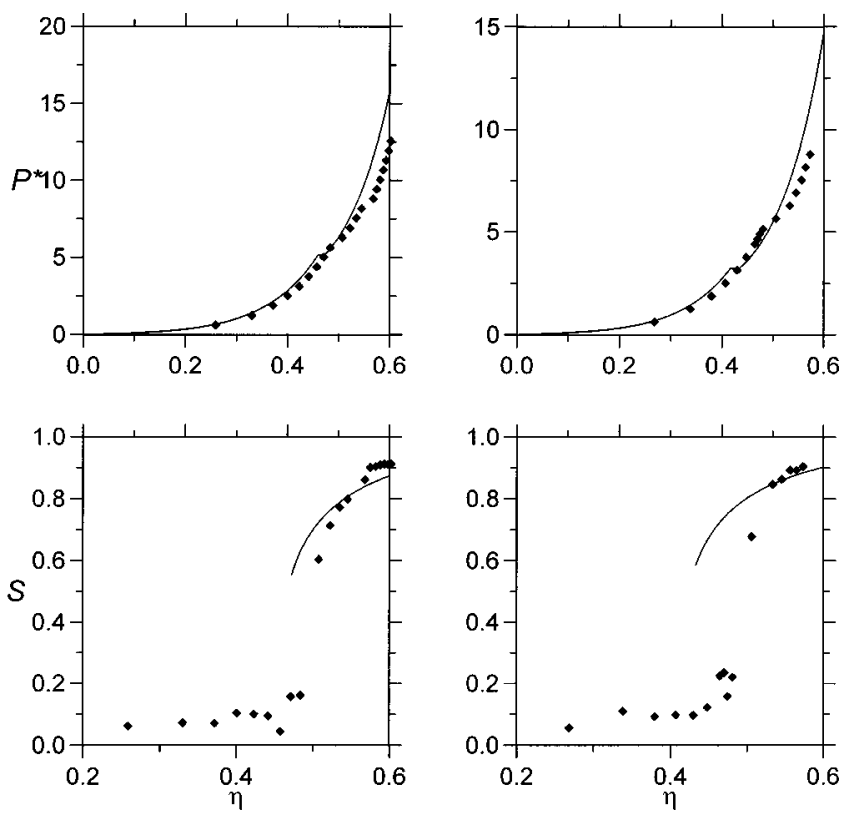

FIG. 5. Same as the Fig. 4, but the values of the reduced dipole moments are $\mu^{*}=1.5$ on the left-hand side and $\mu^{*}=2$ on the right-hand side.

$=1.25$ ) which is above the critical point. The density dependence of the pressure of the dipolar Gay-Berne model is seen to be well predicted, at least for low values of the dipole moment. In line with the molecular simulation studies there is no indication of ferroelectric nematic ordering, and only the usual isotropic-nematic transition is seen. The results for the isotropic-nematic phase equilibria are gathered in Table I together with the existing simulation data. ${ }^{52,53}$ The agreement is quite good for reduced dipole moments up to a value of $\mu^{*}=1$. For stronger dipole moments, the theory predicts much lower transition pressures and densities than the simulated values. According to the simulations, the isotropicnematic transition densities are not affected significantly by the dipolar interaction (apart from a very slight destabilization to higher densities), while the theoretical predictions suggest that the transition is shifted to lower densities with increasing dipole moment. This can be attributed to the approximation used for the dipolar contribution to the free energy [Eq. (18)] in which the virial series is truncated after the second virial coefficient term. The same is found for the

TABLE I. Isotropic-nematic phase coexistence data of dipolar Gay-Berne fluid obtained by Monte Carlo (MC) simulations (Refs. 52 and 53) and from the present theory at $T^{*}=1.25$ and $\kappa^{\prime}=5 . \eta_{I}$ and $\eta_{N}$ denote the packing fraction of isotropic and nematic phases, respectively; $P^{*}=\beta P / v_{0}$ and $\mu^{*}=\sqrt{\mu^{2} / \varepsilon_{s s} \sigma_{s s}^{3}}$.

\begin{tabular}{lcccc}
\hline \hline & $\eta_{I}$ & $\eta_{N}$ & $P_{I N}^{*}$ & $\mu^{*}$ \\
\hline MC & 0.477 & 0.501 & 6.27 & 0.5 \\
Theory & 0.475 & 0.487 & 6.15 & 0.5 \\
MC & 0.479 & 0.503 & 6.28 & 1.0 \\
Theory & 0.473 & 0.484 & 5.96 & 1.0 \\
MC & 0.482 & 0.506 & 6.28 & 1.5 \\
Theory & 0.459 & 0.471 & 5.16 & 1.5 \\
MC & 0.479 & 0.504 & 5.64 & 2.0 \\
Theory & 0.416 & 0.432 & 3.21 & 2.0 \\
\hline \hline
\end{tabular}


other theoretical studies of dipolar hard spherocylinders at this level of approximation. ${ }^{91,99,110}$ It is evident that higher order terms should be incorporated to improve the description of systems with larger dipole moments (e.g., see Refs. 114 and 115). This relative insensitivity of the isotropicnematic transition to the dipolar interactions has been discussed in terms of the dimerisation of the molecules for strong dipolar interactions (implicitly incorporating "threebody" interactions). ${ }^{116,117}$ We will, therefore, only discuss the dependence of the phase diagram on the molecular shape for the dipolar Gay-Berne system with a relatively weak dipole moment of $\mu^{*}=0.5$. Before we turn to the effect of molecular shape it is important to mention that the dipolar GayBerne system also exhibits a nematic-smectic A phase transition at higher densities; the treatment of layering transitions to smectic phases or of solid phases has not been incorporated in the present theory.

A change in the shape of the molecule from prolate to oblate has a profound effect on the phase behavior of dipolar Gay-Berne fluids as shown in Fig. 6. For a shape anisotropy of $\kappa=3$ corresponding to a prolate molecule, only vapor, liquid, and nematic phases are found. The first-order isotropic-nematic biphasic region widens with decreasing temperature until it meets the vapor-liquid coexitence curve at the vapor-liquid-nematic triple point. Below the triple point, the vapor phase is in coexistence with the nematic phase. This trend is very similar to what has been found for the nonpolar Gay-Berne fluid, with the exception that there is no vapor-liquid-nematic triple point because the smectic B phase preempts the nematic phase at the lower temperatures. ${ }^{27,28}$ As was mentioned earlier, positionally ordered phases cannot be examined with the present theory. The absence of ferroelectric nematic ordering in the prolate dipolar Gay-Berne system is consistent with the findings of the simulations. ${ }^{51-55}$

For lower values of the aspect ratio the extent of the vapor-liquid coexistence region is first seen to increase with an increase in the critical temperature and a decrease in the triple point; at the same time the isotropic-nematic transition is shifted to higher densities where it is probably metastable and preempted by the formation of solid phases. Furthermore, the isotropic-nematic transition becomes less first order. This tendency is observed for molecular aspect ratios up to $\kappa=1.5$, where an additional region of ferroelectric ordering is found at low temperature: a region of vaporferroelectric nematic coexistence is seen at very low temperature, and regions of vapor-liquid and isotropicferroelectric coexistence are seen above the triple point. A second critical point is seen at which the isotropic liquid, nematic, and ferroelectric nematic phases are in coexistence; above this critical temperature, the isotropic-nematic transition is weakly first order while the nematic-ferroelectric nematic transition is second order. One should again note that for this aspect ratio of $\kappa=1.5$ the ferrolectric phase is seen for packing fractions above $50 \%$, which suggests that the polar phase is probably metastable with respect to a solid phase; this was seen for spherical molecules where $\kappa=1 . .^{90}$

For molecules with an oblate shape (an aspect ratio of $\kappa=0.5$ ), a dramatic change in the phase diagram can be seen.
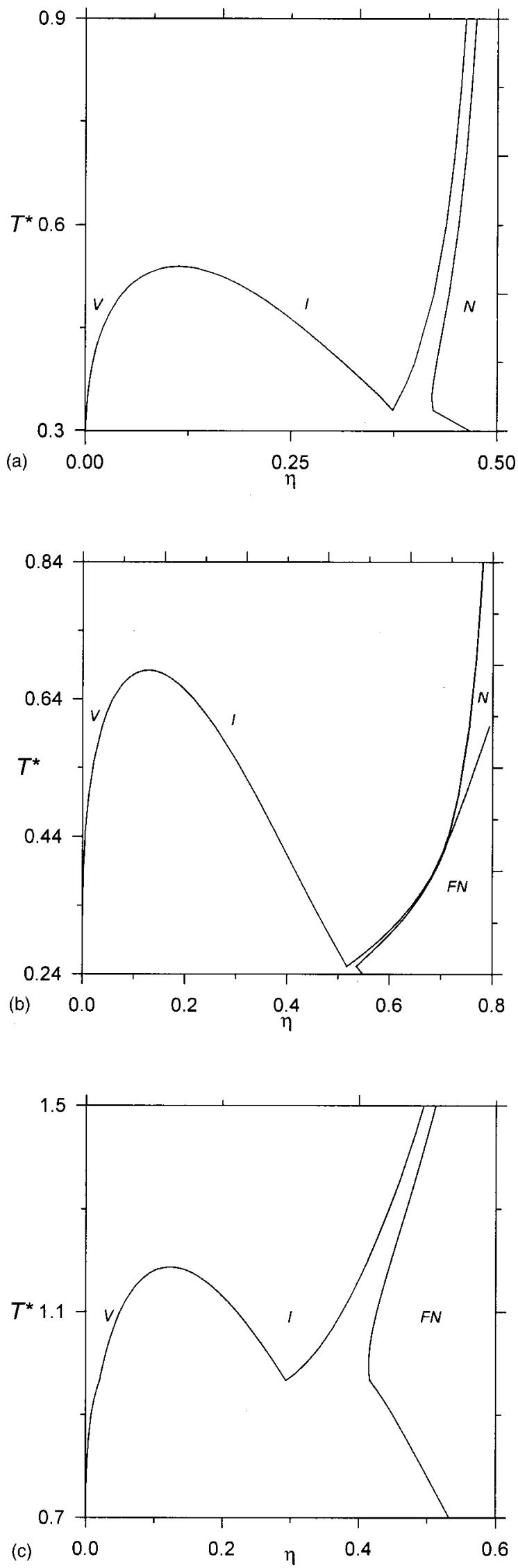

FIG. 6. The effect of molecular elongation on the phase diagram of the dipolar Gay-Berne fluid with an energy depth anisotropy of $\kappa^{\prime}=5$ and a dipole moment of $\mu^{*}=0.5$. The values of the elongation are (a) $\kappa=3$, (b) 1.5 , and (c) 0.5 . The regions of stability of the vapor $(V)$, isotropic $(I)$, nematic $(N)$, and ferroelectric nematic $(F N)$ phases are indicated on the figures. 
The region of the ferroelectric nematic phase is shifted towards lower densities, corresponding to packing fractions of as low as $40 \%$. The relative extent of vapor-liquid coexistence is seen to decrease in spite of the fact that the vaporliquid critical point is at a higher temperature; this is because of a corresponding increase in the temperature of the vaporliquid-ferroelectric triple point. Although not shown in the scale of the figure, the ferroelectric nematic phase remains stable for temperatures as high as $T^{*}=2.7$, where there is a liquid-nematic-ferroelectric nematic critical point (as was seen for the system with an aspect ratio of $\kappa=1.5$ ); above this temperature there is a stable region of the nematic phase. Furthermore, we expect that for more oblate particles the vapor-liquid transition could be totally preempted by isotropic-ferroelectric nematic transition. Having found a ferroelectric nematic phase at relatively high temperature and low densities $(\eta \approx 0.4)$ suggests that ferroelectric nematic ordering is much more likely for oblate-shaped particles than for the prolate molecules. It is hoped that at these relatively low densities the ferroelectric phase found for the oblate polar Gay-Berne molecules will be stable relative to the solid (and possibly even columnar phases) which are expected to be stable above packing fractions of about $50 \%$. It is gratifying to find the possibility of polar phases with relatively weak values of the dipole moment. These findings are in general agreement with the conclusions of Groh and Dietrich, ${ }^{91}$ who studied fluids of dipolar hard ellipsoids, and also with the simulations of Patey an co-workers. ${ }^{71,72}$ Ferroelectric phases were not found for the oblate dipolar GayBerne system at the selected states simulated by Berardi et al. ${ }^{67}$ for a single packing fraction of $\eta \approx 0.45$. The particles were more oblate $(\kappa=0.345)$ and the energy anisotropy was chosen in order to favor the end-to-end configuration $\left(\kappa^{\prime}=1 / 5\right)$ which will tend to stabilize columnar ordering. In the case of our dipolar Gay-Berne system, the ferroelectric phases are found at low density for the less oblate molecules $(\kappa=0.5)$ and we also favor the side-by-side interaction $\left(\kappa^{\prime}=5\right)$, in this way minimizing the possible stability of the columnar phase.

It is useful to examine our results using the analysis of Blinov. ${ }^{79}$ The point at which the polar nematic phase first starts to become stable is when the dipolar interactions are of the order of the kinectic energy, or roughly speaking when $\mu^{2} / \nu_{m} \geqslant k T$, where $\nu_{m}$ is the molecular volume. In the case of the HGO model $\nu_{m}=\pi \kappa \sigma_{s s}^{3} / 6$ so in our reduced units we find that one can expect a ferroelectric phase for temperatures below $T^{*} \leqslant 6 \mu^{* 2} /(\pi \kappa)$. For prolate molecules with $\kappa=3$, this corresponds to a very low temperature of $T^{*}$ $\leqslant 0.16$ which is clearly in the solid part of the phase diagram [see Fig. 6(a)]. When the shape anisotropy is reduced to $\kappa$ $=1.5$, the value of $T^{*} \leqslant 0.32$ indicates the limit of stability of the polar phase, which is not far from our theoretical prediction for the temperature of the vapor-liquid-ferroelectric triple point [see Fig. 6(b)]. For oblate shaped molecules with $\kappa=0.5$, the rough relation predicts a much higher temperature of $T^{*} \leqslant 0.96$ for the limit of stability of the ferroelectric nematic phase, which again is not far from our predictions for the triple point temperature [see Fig. 6(c)].

\section{CONCLUSIONS}

A simple version of the classical density-functional theory is presented for systems of particles interacting via a complex intermolecular potential which incorporates anisotropic repulsive, dispersive, and polar interactions. The theory is only developed to deal with spatially homogeneous phases; only orientational ordering in fluid phases are considered. Positional ordering into smectic layers or columnar phases could also be treated, e.g., see the work of Velasco and Mederos, ${ }^{49}$ but this is not straightforward for potentials such as the Gay-Berne model and is beyond the scope of the present study. We thus focus on regions of the phase behavior where only spatially homogeneous fluid phases are expected. By combining a decoupling approximation of the repulsive forces with a simple mean-field treatment of the dispersive forces developed for the Lennard-Jones potential, a tractable yet reasonably quantitative theory for the GayBerne model is obtained. The approach is then extended to the dipolar Gay-Berne model by using a perturbation theory at the second virial level; a proper treatment of the shape independent thermodynamic limit for a system with homogeneous magnetization is used. The theory provided a good description of the equation of state and the order parameters in the ordered phases for the dipolar-Gay-Berne system; we turn our attention to systems with moderate densities and low dipole moments, where the theory is expected to give the best description. In contrast to the findings of simulation studies, the dipolar interactions are predicted to stabilize the nematic phase relative to the isotropic; this was also seen in other theoretical studies at the second-virial level. ${ }^{91,99,110}$ As was mentioned earlier higher body terms have to be incorporated to reproduce the relative insensitivity of the isotropicnematic transition to the dipolar interactions (e.g., see Refs. 114 and 115); unfortunately the incorporation of the threebody terms is not trivial for the Gay-Berne model. Ferroelectric nematic ordering is predicted to be unlikely in dipolar molecules of prolate or spherical shape; the polar fluid phase is most likely preempted by solidification. Probably the most important single result of our study is that we have found a relatively low-density high-temperature region in the phase diagram of oblate Gay-Berne molecules with a central longitudinal dipole where a ferroelectric phase is possible; the relatively low-density of this polar phase would mean that it preempts columnar ordering. It is hoped that our results will be used as a guide in future simulation studies of oblate dipolar Gay-Berne molecules to ascertain the stability of a ferroelectic nematic phase in such a system.

\section{ACKNOWLEDGMENTS}

S.V. would like to thank the ROPA program of the Engineering and Physical Sciences Research Council (EPSRC) for a research fellowship (GR/N03358). The authors acknowledge support from the Joint Research Equipment Initiative (JREI) of the EPSRC for computer hardware (GR/ M94427), the Royal Society-Wolfson Foundation for the award of a refurbishment grant, and the Hungarian National Research Fund (OTKA-TO29327). 
${ }^{1}$ L. Onsager, Phys. Rev. 62, 558 (1942); L. Onsager, Ann. N.Y. Acad. Sci. 51, 627 (1949)

${ }^{2}$ D. Frenkel and B. M. Mulder, Mol. Phys. 55, 1171 (1985).

${ }^{3}$ M. P. Allen and C. P. Mason, Mol. Phys. 86, 467 (1995).

${ }^{4}$ D. Frenkel, J. Phys. Chem. 92, 3280 (1988).

${ }^{5}$ D. Frenkel, H. N. W. Lekkerkerker, and A. Stroobants, Nature (London) 332, 822 (1988).

${ }^{6}$ S. C. McGrother, D. C. Williamson, and G. Jackson, J. Chem. Phys. 104, 6755 (1996)

${ }^{7}$ P. Bolhuis and D. Frenkel, J. Chem. Phys. 106, 666 (1997).

${ }^{8}$ P. I. C. Teixeira, J. M. Tavares, and M. M. Telo da Gama, J. Phys.: Condens. Matter 12, R411 (2000).

${ }^{9}$ S. C. McGrother, A. Gil-Villegas, and G. Jackson, Mol. Phys. 95, 657 (1998).

${ }^{10}$ J. G. Gay and B. J. Berne, J. Chem. Phys. 74, 3316 (1981).

${ }^{11}$ D. J. Adams, G. R. Luckhurst, and R. W. Phippen, Mol. Phys. 61, 1575 (1987).

${ }^{12}$ G. R. Luckhurst, R. A. Stephens, and R. W. Phippen, Liq. Cryst. 8, 451 (1990).

${ }^{13}$ E. de Miguel, L. F. Rull, M. K. Chalam, and K. E. Gubbins, Mol. Phys. 71, 1223 (1990).

${ }^{14}$ E. de Miguel, L. F. Rull, M. K. Chalam, K. E. Gubbins, and F. van Swol, Mol. Phys. 72, 593 (1991).

${ }^{15}$ M. K. Chalam, K. E. Gubbins, E. de Miguel, and L. F. Rull, Mol. Simul. 7, 357 (1991).

${ }^{16}$ E. de Miguel, L. F. Rull, and K. E. Gubbins, Physica A 177, 174 (1991).

${ }^{17}$ E. de Miguel, L. F. Rull, M. K. Chalam, and K. E. Gubbins, Mol. Phys. 74, 405 (1991)

${ }^{18}$ E. de Miguel, L. F. Rull, and K. E. Gubbins, Phys. Rev. A 45, 3813 (1992).

${ }^{19}$ R. Berardi, A. P. J. Emerson, C. Zannoni, J. Chem. Soc., Faraday Trans. 89, 4069 (1993).

${ }^{20}$ G. R. Luckhurst and P. S. J. Simmonds, Mol. Phys. 80, 233 (1993).

${ }^{21}$ S. S. Sarman, P. T. Cummings, and D. J. Evans, Int. J. Thermophys. 15, 1125 (1994)

${ }^{22}$ A. M. Smondyrev, G. B. Loriot, and R. A. Pelcovits, Phys. Rev. Lett. 75, 2340 (1995)

${ }^{23}$ L. F. Rull, Physica A 220, 113 (1995)

${ }^{24}$ R. Hashim, G. R. Luckhurst, and S. Romano, J. Chem. Soc., Faraday Trans. 91, 2141 (1995).

${ }^{25}$ M. P. Allen, J. T. Brown, and M. A. Warren, J. Phys.: Condens. Matter 8, 9433 (1996)

${ }^{26}$ M. P. Allen, M. A. Warren, A. Sauron, and W. Smith, J. Chem. Phys. 105, 2850 (1996)

${ }^{27}$ E. de Miguel, E. M. del Rio, J. T. Brown, and M. P. Allen, J. Chem. Phys. 105, 4234 (1996).

${ }^{28}$ J. T. Brown, M. P. Allen, E. M. del Rio, and E. de Miguel, Phys. Rev. E 57, 6685 (1998).

${ }^{29}$ S. Cozzini, L. F. Rull, G. Ciccoti, and G. V. Paolini, Physica A 240, 173 (1997).

${ }^{30}$ M. A. Bates and G. R. Luckhurst, J. Chem. Phys. 110, 7087 (1999).

${ }^{31}$ E. de Miguel, Molec. Phys. (in press, 2002).

${ }^{32}$ A. P. J. Emerson, G. R. Luckhurst, and S. G. Whatling, Mol. Phys. 82, 113 (1994).

${ }^{33}$ M. D. Deluca, M. P. Neal, and C. M. Care, Liq. Cryst. 16, 257 (1994).

${ }^{34}$ M. D. Deluca, M. K. Griffiths, C. M. Care, and M. P. Neal, Int. J. Electron. 77, 907 (1994).

${ }^{35}$ M. P. Neal, M. D. Deluca, and C. M. Care, Mol. Simul. 14, 245 (1995).

${ }^{36}$ M. A. Bates and G. R. Luckhurst, J. Chem. Phys. 104, 6696 (1996).

${ }^{37}$ J. Stelzer, M. A. Bates, L. Longa, and G. R. Luckhurst, J. Chem. Phys. 107, 7483 (1997).

${ }^{38}$ E. M. del Rio, E. de Miguel, and L. F. Rull, Physica A 213, 138 (1995).

${ }^{39}$ A. P. J. Emerson, S. Faetti, and C. Zannoni, Chem. Phys. Lett. 271, 241 (1997).

${ }^{40}$ M. A. Bates and C. Zannoni, Chem. Phys. Lett. 280, 40 (1997).

${ }^{41}$ S. J. Mills, C. M. Care, M. P. Neal, and D. J. Cleaver, Phys. Rev. E 58, 3284 (1998)

${ }^{42}$ E. de Miguel, and E. M. del Rio, Int. J. Mod. Phys. C 10, 431 (1999).

${ }^{43}$ S. J. Mills, C. M. Care, M. P. Neal, M. R. Wilson, M. P. Allen, and D. J. Cleaver, J. Mol. Liq. 85, 185 (2000).

${ }^{44}$ B. Tjipto-Margo and D. E. Sullivan, J. Chem. Phys. 88, 6620 (1988).

${ }^{45}$ E. T. Brooklevinson, A. V. Zakharov, and V. B. Nemtsov, J. Phys. I 2, 2075 (1992).

${ }^{46}$ E. Velasco, A. M. Somoza, and L. Mederos, J. Chem. Phys. 102, 8107 (1995).
${ }^{47}$ V. V. Ginzburg, M. A. Glaser, and N. A. Clark, Liq. Cryst. 21, 265 (1996).

${ }^{48}$ V. V. Ginzburg, M. A. Glaser, and N. A. Clark, Liq. Cryst. 23, 227 (1997).

${ }^{49}$ E. Velasco and L. Mederos, J. Chem. Phys. 109, 2361 (1998).

${ }^{50} \mathrm{~W}$. L. Wagner and L. Bennett, Mol. Phys. 94, 571 (1998).

${ }^{51}$ K. Satoh, S. Mita, and S. Kondo, Liq. Cryst. 20, 757 (1996).

${ }^{52}$ M. Houssa, A. Oualid, and L. F. Rull, Mol. Phys. 94, 439 (1998).

${ }^{53}$ M. Houssa, L. F. Rull, and S. C. McGrother, J. Chem. Phys. 109, 9529 (1998).

${ }^{54}$ M. Houssa, L. F. Rull, and S. C. McGrother, Int. J. Mod. Phys. C 10, 391 (1999).

${ }^{55}$ M. Houssa, S. C. McGrother, and L. F. Rull, Comput. Phys. Commun. 122, 259 (1999)

${ }^{56}$ K. Satoh, S. Mita, and S. Kondo, Chem. Phys. Lett. 255, 99 (1996).

${ }^{57}$ R. Berardi, S. Orlandi, and C. Zannoni, Chem. Phys. Lett. 261, 357 (1996).

${ }^{58}$ K. Satoh, S. Mita, and S. Kondo, Molec. Cryst. Liq. Cryst. 300, 143 (1997)

${ }^{59}$ S. C. McGrother, A. Gil-Villegas, and G. Jackson, J. Phys.: Condens. Matter 8, 9649 (1996).

${ }^{60}$ E. Gwozdz, A. Brodka, and K. Pasterny, Chem. Phys. Lett. 267, 557 (1997)

${ }^{61}$ E. Gwozdz, A. Brodka, and K. Pasterny, J. Mol. Struct. 450, 1 (1998).

${ }^{62}$ R. Berardi, S. Orlandi, and C. Zannoni, Int. J. Mod. Phys. C 10, 477 (1999).

${ }^{63}$ K. Pasterny, E. Gwozdz, and A. Brodka, J. Mol. Liq. 85, 173 (2000).

${ }^{64}$ E. Gwozdz, A. Brodka, and K. Pasterny, J. Mol. Struct. 555, 257 (2000).

${ }^{65}$ E. Gwozdz and A. Brodka, Acta Phys. Pol. A 98, 645 (2000).

${ }^{66}$ A. Gil-Villegas, S. C. McGrother, and G. Jackson, Chem. Phys. Lett. 269, 441 (1997).

${ }^{67}$ R. Berardi, S. Orlandi, and C. Zannoni, J. Chem. Soc., Faraday Trans. 93, 1493 (1997).

${ }^{68}$ R. Berardi, S. Orlandi, and C. Zannoni, Phys. Chem. Chem. Phys. 2, 2933 (2000).

${ }^{69}$ J.-J. Weis, D. Levesque, and G. J. Zarragoicoechea, Phys. Rev. Lett. 69, 913 (1992).

${ }^{70}$ G. J. Zarragoicoechea, D. Levesque, and J.-J. Weis, Mol. Phys. 78, 1475 (1993).

${ }^{71}$ G. Ayton and G. N. Patey, Phys. Rev. Lett. 76, 239 (1996).

${ }^{72}$ G. Ayton, D. Q. Wei, and G. N. Patey, Phys. Rev. E 55, 447 (1997).

${ }^{73}$ D. Q. Wei and G. N. Patey, Phys. Rev. Lett. 68, 2043 (1992).

${ }^{74}$ D. Q. Wei and G. N. Patey, Phys. Rev. A 46, 7783 (1992).

${ }^{75}$ J.-J. Weis, D. Levesque, and G. J. Zarragoicoechea, Phys. Rev. E 48, 3728 (1993).

${ }^{76}$ A. V. Zakharov and S. Romano, Phys. Rev. E 58, 7428 (1998).

${ }^{77}$ A. V. Zakharov, S. Romano, and A. Maliniak, Phys. Rev. E 60, R1142 (1999).

${ }^{78}$ H. Takezoe and J. Watanabe, Molec. Cryst. Liq. Cryst. 328, 325 (1999).

${ }^{79}$ L. M. Blinov, Liq. Cryst. 24, 143 (1998)

${ }^{80}$ H. E. Stanley, Introduction to Phase Transitions and Critical Phenomena (Oxford University Press, 1987).

${ }^{81}$ P. Palffy-Muhoray, M. A. Lee, and R. G. Petschek, Phys. Rev. Lett. 60, 2303 (1988)

${ }^{82}$ B. Park, J. W. Wu, and H. Takezoe, Phys. Rev. E 63, 021707 (2001).

${ }^{83}$ M. Baus and J.-L. Colot, Phys. Rev. A 40, 5444 (1989).

${ }^{84}$ A. Perera and G. N. Patey, J. Chem. Phys. 91, 3045 (1989).

${ }^{85}$ D. Q. Wei, G. N. Patey, and A. Perera, Phys. Rev. E 47, 506 (1993).

${ }^{86}$ J. Lee and S. D. Lee, Molec. Cryst. Liq. Cryst. 254, 395 (1994).

${ }^{87}$ B. Groh and S. Dietrich, Phys. Rev. Lett. 72, 2422 (1994).

${ }^{88}$ B. Groh and S. Dietrich, Phys. Rev. E 50, 3814 (1994).

${ }^{89}$ B. Groh and S. Dietrich, Phys. Rev. E 53, 2509 (1996).

${ }^{90}$ B. Groh and S. Dietrich, Phys. Rev. E 54, 1687 (1996).

${ }^{91}$ B. Groh and S. Dietrich, Phys. Rev. E 55, 2892 (1997).

${ }^{92}$ B. Groh and S. Dietrich, Phys. Rev. E 57, 4535 (1998).

${ }^{93}$ S. T. Lagerwall, J. Phys.: Condens. Matter 8, 9143 (1996).

${ }^{94}$ J. Weeks, D. Chandler, and H. C. Andersen, J. Chem. Phys. 54, 5237 (1971).

${ }^{95}$ G. Vroege and H. N. W. Lekkerkerker, Recent Progr. Phys. 55, 1241 (1992).

${ }^{96}$ M. A. Cotter, J. Chem. Phys. 66, 1098 (1977).

${ }^{97}$ B. Barboy and W. M. Gelbart, J. Chem. Phys. 71, 3053 (1979).

${ }^{98}$ A. Samborski, G. T. Evans, C. P. Mason, and M. P. Allen, Mol. Phys. 81, 263 (1994).

${ }^{99}$ C. Vega and S. Lago, J. Chem. Phys. 100, 6727 (1994).

${ }^{100}$ P. Tarazona, Philos. Trans. R. Soc. London, Ser. A 344, 307 (1993). 
${ }^{101}$ J. D. Parsons, Phys. Rev. A 19, 1225 (1979).

${ }^{102}$ S.-D. Lee, J. Chem. Phys. 87, 4972 (1987).

${ }^{103}$ N. F. Carnahan and K. E. Starling, J. Chem. Phys. 51, 635 (1969).

${ }^{104}$ B. J. Berne and P. Pechukas, J. Chem. Phys. 56, 4213 (1972).

${ }^{105}$ T. Boublik and I. Nezbeda, Collect. Czech. Chem. Commun. 51, 2301 (1986).

${ }^{106}$ J. P. Noworyta, D. Henderson, and S. Sokolowsky, Mol. Phys. 96, 1139 (1999).

${ }^{107}$ S. Varga, D. Boda, D. Henderson, and S. Sokolowski, J. Colloid Interface Sci. 227, 223 (2000).

${ }^{108}$ P. I. Teixeira and M. M. Telo da Gama, J. Phys.: Condens. Matter 3, 111 (1991)

${ }^{109}$ P. Frodl and S. Dietrich, Phys. Rev. A 45, 7330 (1992).
${ }^{110}$ A. G. Vanakaras and D. J. Photinos, Mol. Phys. 85, 1089 (1995).

${ }^{111}$ J. Herzfield, A. E. Berger, and J. W. Wingate, Macromolecules 17, 1718 (1984).

${ }^{112}$ W. H. Press, B. P. Flannery, S. A. Teukolsly, and W. T. Vetterling, $\mathrm{Nu}-$ merical Recipes (Cambridge University Press, Cambridge, 1996).

${ }^{113}$ B. Smit, C. P. Williams, E. M. Hendriks, and S. W. De Leeuw, Mol. Phys. 68, 765 (1989).

${ }^{114}$ S. C. McGrother, G. Jackson, and D. J. Photinos, Mol. Phys. 91, 751 (1997).

${ }^{115}$ D. C. Williamson and F. Del Rio, J. Chem. Phys. 107, 9549 (1997).

${ }^{116}$ A. V. Emelyanenko and M. A. Osipov, Liq. Cryst. 26, 187 (1999).

${ }^{117}$ A. V. Emelyanenko and M. A. Osipov, Crystallogr. Rep. 45, 501 (2000). 